\title{
Expression of one important chaperone protein, heat shock protein 27 , in neurodegenerative diseases
}

Xuekai Zhang ${ }^{1,2}$, Jing Shi ${ }^{1}$, Jinzhou Tian ${ }^{1,3^{*}}$, Andrew C Robinson ${ }^{2}$, Yvonne S Davidson ${ }^{2}$ and David M Mann ${ }^{2,4^{*}}$

\begin{abstract}
Introduction: Many neurodegenerative diseases are characterised by accumulations of misfolded proteins that can colocalise with chaperone proteins (for example, heat shock protein 27 (HSP27)), which might act as modulators of protein aggregation.
\end{abstract}

Methods: The role of HSP27 in the pathogenesis of neurodegenerative disorders such as frontotemporal lobar degeneration (FTLD), Alzheimer's disease (AD) and motor neuron disease (MND) was investigated. We used immunohistochemical and Western blot analysis to determine the distribution and amount of this protein in the frontal and temporal cortices of diseased and control subjects.

Results: HSP27 immunostaining presented as accumulations of granules within neuronal and glial cell perikarya. Patients with AD and FTLD were affected more often, and showed greater immunostaining for HSP27, than patients with MND and controls. In FTLD, there was no association between HSP27 and histological type. The neuropathological changes of FTLD, AD and MND were not immunoreactive to HSP27. Western blot analysis revealed higher HSP27 expression in FTLD than in controls, but without qualitative differences in banding patterns.

Conclusions: The pattern of HSP27 immunostaining observed may reflect the extent of ongoing neurodegeneration in affected brain areas and is not specific to FTLD, AD or MND. It may represent an accumulation of misfolded, damaged or unwanted proteins, awaiting or undergoing degradation.

\section{Introduction}

Many neurodegenerative diseases, also termed as protein conformational diseases [1], are characterised by accumulations of misfolded proteins that often share morphological and biochemical features and can colocalise with several other proteins, including various chaperone proteins. The accumulation of misfolded proteins may adversely affect neuronal connectivity and plasticity and trigger cell death signalling pathways [2]. These misfolded proteins include amyloid- $\beta$ protein $(A \beta)$ and tau in Alzheimer's disease (AD); $\alpha$-synuclein and synphilin 1 in Parkinson's disease (PD); polyglutamine (polyQ)-

\footnotetext{
* Correspondence: jztian@hotmail.com; david.mann@manchester.ac.uk 'The Third Department of Neurology, Dongzhimen Hospital, Beijing University of Chinese Medicine, No. 5 Haiyuncang Street, Dongcheng District, Beijing 100700, China

${ }^{2}$ Clinical and Cognitive Sciences Research Group, Institute of Brain, Behaviour and Mental Health, Faculty of Medical and Human Sciences, University of Manchester, Salford Royal Hospital, Salford M6 8HD, UK

Full list of author information is available at the end of the article
}

expanded huntingtin in Huntington's disease; transactive response DNA binding protein 43 (TDP-43) and copper-zinc superoxide dismutase 1 in motor neuron disease (MND); and tau, TDP-43 and fused in sarcoma in frontotemporal lobar degeneration (FTLD). These aggregates may consist of oligomeric complexes of non-native secondary structures and demonstrate poor solubility in aqueous or detergent solvents [3]. Chaperone proteins, such as heat shock proteins (HSPs), play a prime role in protein homeostasis by binding to substrates at risk, thereby keeping them in a state competent for either refolding or degradation [4]. Chaperone proteins have therefore been implicated as potent modulators of protein conformational disorders, suppressing toxicity of misfolding proteins and modifying early events in the aggregation process in a cooperative and sequential manner reminiscent of their functions in de novo protein folding $[5,6]$.

HSP27, also known as HSPB1, is one of the best studied members of the small HSP family. It functions as a 
molecular chaperone, aiding the refolding of nonnative proteins, and plays a critical role in stabilisation of the cytoskeleton through interactions with several cytoskeletal components, such as actin, intermediate filaments and microtubules [7]. Normally, when functioning as a molecular chaperone, HSP27 can bind non-native substrates in an ATP-independent manner, which is quite different from most HSPs [8]. However, for substrate release, it requires the help of ATP-dependent chaperone proteins, such as HSP70 and HSP104 [9]. Additionally, HSP27 can exhibit a number of cytoprotective properties in cell culture, attenuating apoptosis through interaction with proapoptotic proteins such as Daxx, cytosolic cytochrome $c$ and caspase 3 , as well as suppressing aggregate formation $[10,11]$.

HSP27 has been implicated in various neurodegenerative diseases. For example, a highly induced expression of HSP27 has been reported in the brains of aged persons and of patients with $\mathrm{AD}$, being present in proliferating astrocytes, especially in AD in those areas rich in senile (A $\beta$ ) plaques. Neurofibrillary tangles, Hirano bodies and some hippocampal neurons have also been reported to be HSP27-immunoreactive. However, in control brains, HSP27 immunoreactivity was restricted to blood vessels and to occasional astrocytes in the white matter. Similarly, patients with other types of dementia (Parkinsondementia complex, multi-infarct dementia and normal pressure hydrocephalus) showed less expression of HSP27 in reactive astrocytes than that in $\mathrm{AD}$, but more than that in controls. Occasional immunoreactive HSP27 astrocytes have also been reported to be present in Parkinson disease [12]

Although the physiological relationship between HSP27 and misfolded proteins in neurodegenerative diseases has been studied intensively in vivo and in vitro, any potential role of HSP27 in the pathogenesis of neurodegenerative diseases, and especially FTLD, is still incompletely understood. The principal aim of this study was to investigate the possible role of HSP27 in the pathogenesis of FTLD. This was achieved by comparing the distribution and amount of HSP27 in FTLD, AD, MND and control groups by immunohistochemistry, and by characterising biochemical changes using Western blotting.

\section{Methods}

\section{Patients}

The study group comprised 175 patients, composed of 72 patients with FTLD, 46 with AD, 25 with MND and 32 controls (see Table 1 for demographic characteristics). Patients with FTLD fulfilled the Lund-Manchester criteria [13] for sporadic or familial FTLD and their characteristics were consistent with recent consensus criteria [14]. All had been longitudinally assessed within the Cerebral Function Unit, Salford Royal Hospital, with the Manchester Neuropsychological Test Battery. Data collected on each patient included sex, age at diagnosis and at death, and clinical diagnosis. The clinical diagnosis was confirmed at autopsy [15]. The FTLD patients were histologically classified according to the harmonised classification system for FTLD-TDP [16] into FTLD-TDP type A, FTLD-TDP type $\mathrm{B}$ and FTLD-TDP type $\mathrm{C}$ (Table 1 ). The diagnosis of clinical MND (in patients with or without FTLD) was in keeping with El Escorial criteria [17]. Diagnosis of possible or probable $\mathrm{AD}$ was made in accordance with the National Institute of Neurological and Communicative Disorders and Stroke-Alzheimer's Disease and Related Disorders Association criteria [18] and was confirmed pathologically according to Consortium to Establish a Registry for Alzheimer's disease criteria [19]. Control subjects were obtained from a longitudinal ageing cohort of elderly people without clinical symptoms of dementia. All brains were drawn from the Manchester Brain Bank and had been obtained with full ethical permission of the Manchester Brain Bank Management Committee under Tissue Bank Ethical Agreement 08 conferred by Newcastle and North Tyneside 1 Research Ethics Committee following appropriate consenting procedures by the next of kin.

\section{Immunohistochemistry}

Formalin-fixed, paraffin-embedded sections $(6 \mu \mathrm{m})$ of frontal cortex (Brodmann areas 8/9 (BA8/9)) and/or temporal cortex (BA21/22) with hippocampus (where available; see Table 1) were immunostained for HSP27 using a commercially available rabbit polyclonal HSP27 antibody (ab5579; Abcam, Cambridge, UK). A standard immunostaining procedure was followed [20,21]. Briefly, all deparaffinised sections underwent antigen retrieval in $0.1 \mathrm{M}$ citrate buffer, $\mathrm{pH}$ 6.0, by microwave heating for 10 minutes. To quench endogenous peroxidase activity, slides were incubated in $0.3 \%$ hydrogen peroxide in methanol for 30 minutes. Sections were then incubated overnight (at $4^{\circ} \mathrm{C}$ ) in a diluted blocking serum (normal goat serum) to mask nonspecific binding sites. This was followed by 1 -hour immunostaining with primary polyclonal HSP27 antibody diluted to 1:500. Following three 5-minute washes in phosphate-buffered saline, incubations in biotinylated secondary antibody and avidin-biotin complex were performed for 30 minutes each using a VECTASTAIN ABC kit (Vector Laboratories, Peterborough, UK), The immunoreaction was visualised using 3,3'-diaminobenzidine (Sigma, Poole, Dorset, UK). Sections were lightly counterstained with haematoxylin, dehydrated sequentially and mounted with coverslips using DePeX mounting medium. Further sections were immunostained for LAMP1 (rabbit polyclonal antibody AP1823a; Abgent, San Diego, CA, USA), LCA3 (rabbit polyclonal antibody L8793; Sigma, St Louis, MO, USA), p62 (p62-lck ligand, rabbit polyclonal antibody; BD Biosciences, Oxford, UK) and ubiquitin (rabbit polyclonal antibody Z0458; Dako Cytomation, Ely, UK), employing 
Table 1 Demographic details ${ }^{a}$

\begin{tabular}{|c|c|c|c|c|c|}
\hline \multirow[t]{2}{*}{ Group (n) } & \multirow{2}{*}{$\begin{array}{l}M / F, n \\
\text { (missing data) }\end{array}$} & \multirow{2}{*}{$\begin{array}{l}\text { Age at death, yr } \\
(\text { mean } \pm S D)\end{array}$} & \multicolumn{3}{|c|}{ Sections available, $n$} \\
\hline & & & Frontal & Temporal & Hippocampal \\
\hline Controls (32) & $14 / 18$ & $72.0 \pm 21.0$ & 19 & 32 & 31 \\
\hline$A D(46)$ & $23 / 23$ & $72.0 \pm 6.0$ & 46 & 41 & 41 \\
\hline MND (25) & $20 / 5$ & $64.3 \pm 12.0$ & 17 & 25 & 24 \\
\hline FTLD (72) & $41 / 31$ & $65.6 \pm 9.1$ & 71 & 66 & 60 \\
\hline FTLD-tau (31) & 14/14 (3) & $67.2 \pm 9.6$ & 31 & 25 & 25 \\
\hline MAPT (5) & $2 / 3$ & $62.2 \pm 5.4$ & 5 & 4 & 4 \\
\hline Pick (26) & 12/11 (3) & $68.3 \pm 10.1$ & 26 & 21 & 21 \\
\hline FTLD-TDP (41) & $26 / 15$ & $64.7 \pm 8.7$ & 40 & 41 & 35 \\
\hline Type A (17) & $8 / 9$ & $68.7 \pm 4.2$ & 17 & 17 & 14 \\
\hline Type B (14) & $12 / 2$ & $58.3 \pm 9.0$ & 13 & 14 & 12 \\
\hline Type C (10) & $6 / 4$ & $66.5 \pm 9.4$ & 10 & 10 & 9 \\
\hline
\end{tabular}

${ }^{a} A D$, Alzheimer's disease; FTLD, Frontotemporal lobar degeneration; FTLD-MAPT, Frontotemporal lobar degeneration with mutations in the microtubule-associated protein tau; MND, Motor neuron disease; FTLD-tau Pick, Frontotemporal lobar degeneration tau with Pick bodies; TDP, Transactive response DNA binding protein 43 proteinopathy (subtypes A, B and C according to Mackenzie et al. [16]).

antibodies at dilutions of 1:200, 1:200, 1:100 and 1:750, respectively.

\section{Pathological assessment}

The degree and distribution of HSP27 immunostaining in both white matter and grey matter were scored semiquantitatively in temporal cortex and hippocampus, and/or frontal cortex, on a 5 -point scale $(0=$ absent; $1=$ rare; 2 = mild; 3 = moderate; $4=$ severe) using a Leica DMR microscope (×200 magnification, DC 300 F, 10447115; Leica Microsystems, Milton Keynes, UK). The examiner (XK) was blinded to clinical and pathological diagnosis, as well as to the results of the initial scoring of sections, when reexamining the series for a second and third time. Intraclass correlation coefficient and interrater reliability (Cohen's $\mathrm{k}$ ) testing were used to analyse the consistency of scoring over the three assessments. The results showed that there was substantial agreement for ratings performed at each time and for each area (Tables 2 and 3).

\section{Statistical analysis}

All data were analysed using appropriate parametric and nonparametric statistical tests with the SPSS for Windows statistical software package (release 16.0; IBM SPSS, Chicago, IL, USA). In immunohistochemical analysis, the frequency of immunopositive or immunonegative cases in different brain regions between different groups was assessed using Pearson's $\chi^{2}$ test. The severity of HSP27 immunostaining in different brain regions between different groups was analysed using a nonparametric Kruskal-Wallis test with a post hoc Mann-Whitney test. To guard against false-positive results, given the high number of statistical tests employed, only $P$-values $\leq 0.01$ were considered significant.

\section{Western blot analysis}

Brain tissues from five patients were studied (Table 4). Clinically, three patients had been diagnosed with various histological and genetic forms of FTLD. Two cases served as controls, one without any significant pathology and one with mild AD-type changes. Fresh-frozen frontal cortex tissue was used for sequential extraction of proteins with specific buffers. Sequential extracts of proteins are produced after several protein preparation steps, including whole-cell lysate and other fractions-low salt fraction, Triton X-100 fraction, sarkosyl fraction and urea fraction. These fractions represent a series containing a decreasing solubility of (potentially interesting) proteins.

Using an adapted protocol [22], sequential fractions were examined by Western blotting for HSP27. Equal volumes $(25 \mu \mathrm{l})$ of diluted fractions from different samples and $6-\mu \mathrm{l}$ molecular weight marker/ladder were condensed by $5 \%$ stacking gel and resolved by $10 \%$ SDS-PAGE. Electrophoresis was performed at around $120 \mathrm{~V}$ for 60 minutes using a PowerPac Basic power supply (Bio-Rad Laboratories, Hercules, CA, USA). Once the blue line produced by loading buffer $(2 \times$ Laemmli sample buffer) had reached the bottom plastic plate, the electrophoresis was stopped. The proteins on the gel were then transferred to nitrocellulose membranes (Amersham Hybond-ECL; GE Healthcare Life Sciences, Little Chalfont, UK) via a 'semidry' method (Trans-Blot SD Semi-Dry Transfer Cell; Bio-Rad Laboratories), which employs a PowerPac HC Power Supply (Bio-Rad Laboratories) running at $15 \mathrm{~V}$ for 1 hour. Following transfer, membranes were blocked with Tris-buffered saline containing 3\% powered milk for 20 to 60 minutes at room temperature and then probed with rabbit polyclonal HSP27 antibody (ab5579; Abcam), which was diluted to $1: 3,000$ in $3 \%$ bovine serum albumin 
Table 2 Analysis of assessment scores by intraclass correlation coefficients ${ }^{a}$

\begin{tabular}{|c|c|c|c|c|}
\hline & 1st vs 2 nd assessments & 1st vs 3rd assessments & 2nd vs 3rd assessments & 1st vs 2 nd vs 3 rd assessments \\
\hline \multicolumn{5}{|l|}{ Neurons } \\
\hline ICC & 0.874 & 0.815 & 0.854 & 0.892 \\
\hline$P$-value & 0.000 & 0.000 & 0.000 & 0.000 \\
\hline \multicolumn{5}{|c|}{ Grey matter glial cells } \\
\hline ICC & 0.930 & 0.933 & 0.935 & 0.954 \\
\hline$P$-value & 0.000 & 0.000 & 0.000 & 0.000 \\
\hline \multicolumn{5}{|c|}{ White matter glial cells } \\
\hline ICC & 0.958 & 0.928 & 0.935 & 0.960 \\
\hline$P$-value & 0.000 & 0.000 & 0.000 & 0.000 \\
\hline
\end{tabular}

${ }^{a}$ ICC, Intraclass correlation coefficient. Results with perfect agreement $(>0.8)$ are in boldface.

solution with sodium azide overnight at $4^{\circ} \mathrm{C}$. Primary antibodies were detected with horseradish peroxidaseconjugated goat anti-rabbit immunoglobulin G (sc-2004, 1:5,000; Santa Cruz Biotechnology, Santa Cruz, CA, USA), and immunoreactive proteins on the blots were revealed by ECL Plus Western blotting detection reagent ECL Imager system (Thermo Scientific, Rockford, IL USA).

\section{Results}

\section{Cytological observations}

In all cases, HSP27 immunoreactivity was seen in the form of clusters of small or coarse granules within the cytoplasm of neurons and glial cells. This pattern of immunostaining was qualitatively similar in all cases, irrespective of diagnosis, though the severity of changes varied from case to case between diagnostic categories and within each diagnostic category.

In all patients with FTLD and AD (Figures 1 and 2), there were numerous intensely immunostained, HSP27positive granules in the cytoplasm of pyramidal neurons of the cerebral cortex, along with abundant diffuse clusters of similar granules within glial cells in both grey and white matter regions. Such changes were particularly severe in cells of the dentate gyrus and the CA4 region of the hippocampus, Similar, though less severe, changes were seen in these same brain areas in patients with MND and in control subjects.

However, there was no HSP 27 immunostaining of senile plaques or neurofibrillary tangles within the frontal and temporal cortices and the hippocampus in patients with AD, nor was there HSP 27 immunostaining of Pick bodies in patients with FTLD-tau, or TDP- 43 immunoreactive neuronal cytoplasmic inclusions in patients with F TLD-TDP, or MND (not shown).

By immunostaining with LAMP1 antibody, we detected granular structures in neurons and glial cells that were similar in appearance to those visualised by HSP27 immunostaining. However, immunostaining with LCA3 antibody failed to reveal any granular structures or staining patterns similar to those detected with HSP27.

\section{Semiquantitative findings}

The proportion of cases showing HSP27 immunoreactive neurons and/or glial cells in the different brain regions analysed (irrespective of the intensity of staining of individual neurons or glial cells; see below) is shown in

Table 3 Analysis of assessment scores by interrater reliability

\begin{tabular}{|c|c|c|c|}
\hline & 1st vs 2 nd assessments & 1st vs 3rd assessments & 2nd vs 3rd assessments \\
\hline \multicolumn{4}{|l|}{ Neurons } \\
\hline Cohen's K & 0.629 & 0.598 & 0.643 \\
\hline$P$-value & 0.000 & 0.000 & 0.000 \\
\hline \multicolumn{4}{|c|}{ Grey matter glial cells } \\
\hline Cohen's K & 0.444 & 0.671 & 0.666 \\
\hline$P$-value & 0.000 & 0.000 & 0.000 \\
\hline \multicolumn{4}{|c|}{ White matter glial cells } \\
\hline Cohen's K & 0.645 & 0.554 & 0.625 \\
\hline$P$-value & 0.000 & 0.000 & 0.000 \\
\hline
\end{tabular}

Results with substantial agreement (0.61 to 0.80$)$ are in boldface. 
Table 4 Cases assessed in Western blot analysis ${ }^{a}$

\begin{tabular}{llllll}
\hline Case & Onset, $\mathbf{y r}$ & Death, $\mathbf{y r}$ & Duration, $\mathbf{y r}$ & Clinical diagnosis & Pathological diagnosis \\
\hline 1 & 43 & 45 & 2 & FTD + MND & FTLD-TDP B \\
2 & 52 & 65 & 13 & FTD & FTLD-MAPT \\
3 & 63 & 74 & 11 & FTD & FTLD-Pick \\
4 & na & 80 & na & Normal & None \\
5 & na & 82 & na & Normal & Mild AD \\
\hline
\end{tabular}

${ }^{a} A D$, Alzheimer's disease; FTD, Frontotemporal dementia; FTLD, Frontotemporal lobar degeneration; FTLD-Pick, Frontotemporal lobar degeneration tau with Pick bodies; FTLD-MAPT, Frontotemporal lobar degeneration with mutations in the microtubule-associated protein tau; FTLD-TDP B, Frontotemporal lobar degeneration with transactive response DNA binding protein subtype B; MND, Motor neuron disease; na, Not applicable.

Figure 3. The proportion of cases in each diagnostic category showing HSP27 immunoreactive neurons and/or glial cells was highly significantly different between FTLD (overall), AD, MND and control groups for frontal cortex (neurons: $X^{2}=20.8, P=0.001$; glial cells of grey matter: $X^{2}=15.9, P=0.003$; glial cells of white matter: $X^{2}=28.8$, $P=0.000$ ) and temporal cortex (glial cells of grey matter: $X^{2}=15.8, P=0.001$; glial cells of white matter: $X^{2}=23.0$, $P=0.000)$ and marginally significant for temporal cortical neurons $\left(X^{2}=6.8, P=0.080\right)$. However, there were no significant differences between diagnostic groups for the proportion of cases in each diagnostic category showing HSP27 immunoreactive neurons in hippocampus CA4 $\left(X^{2}=5.0, P=0.170\right), C A 3 / 2\left(X^{2}=6.0, P=0.113\right), C A 1$ and subiculum $\left(X^{2}=6.1, P=0.106\right)$ regions or granule cells of the dentate gyrus $\left(\chi^{2}=4.1, P=0.251\right)$, though glial cells of hippocampus did show a significant difference between groups $\left(X^{2}=21.870, P=0.000\right)$.

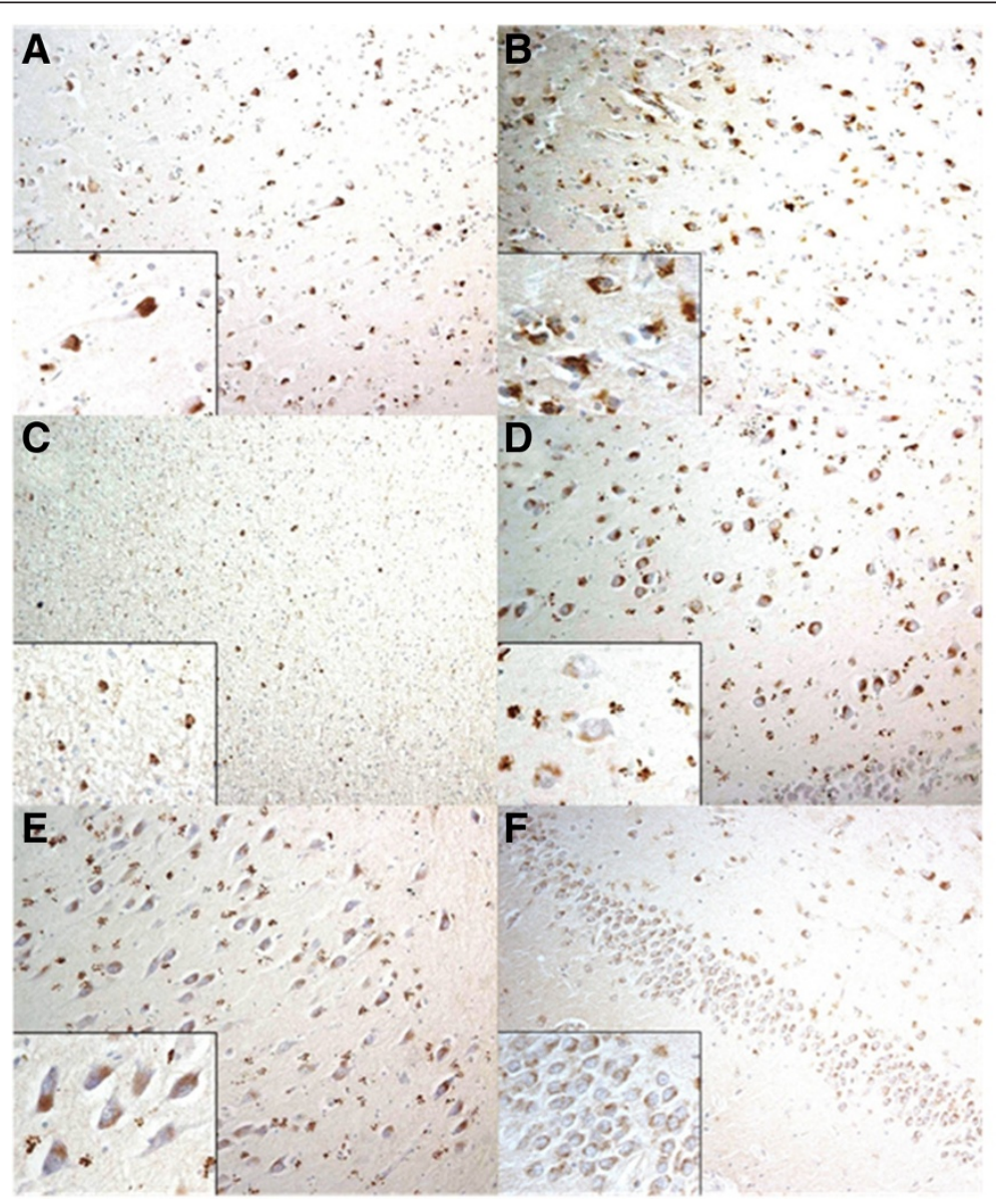

Figure 1 Characteristic HSP27-positive granules in the cytoplasm of neurons and glial cells in different regions of Alzheimer's disease cases under light microscopy. (A) Temporal cortex. (B) Frontal cortex. (C) White matter of cortex. (D) CA4 of hippocampus. (E) CA3 of hippocampus. (F) Dentate gyrus of hippocampus. 


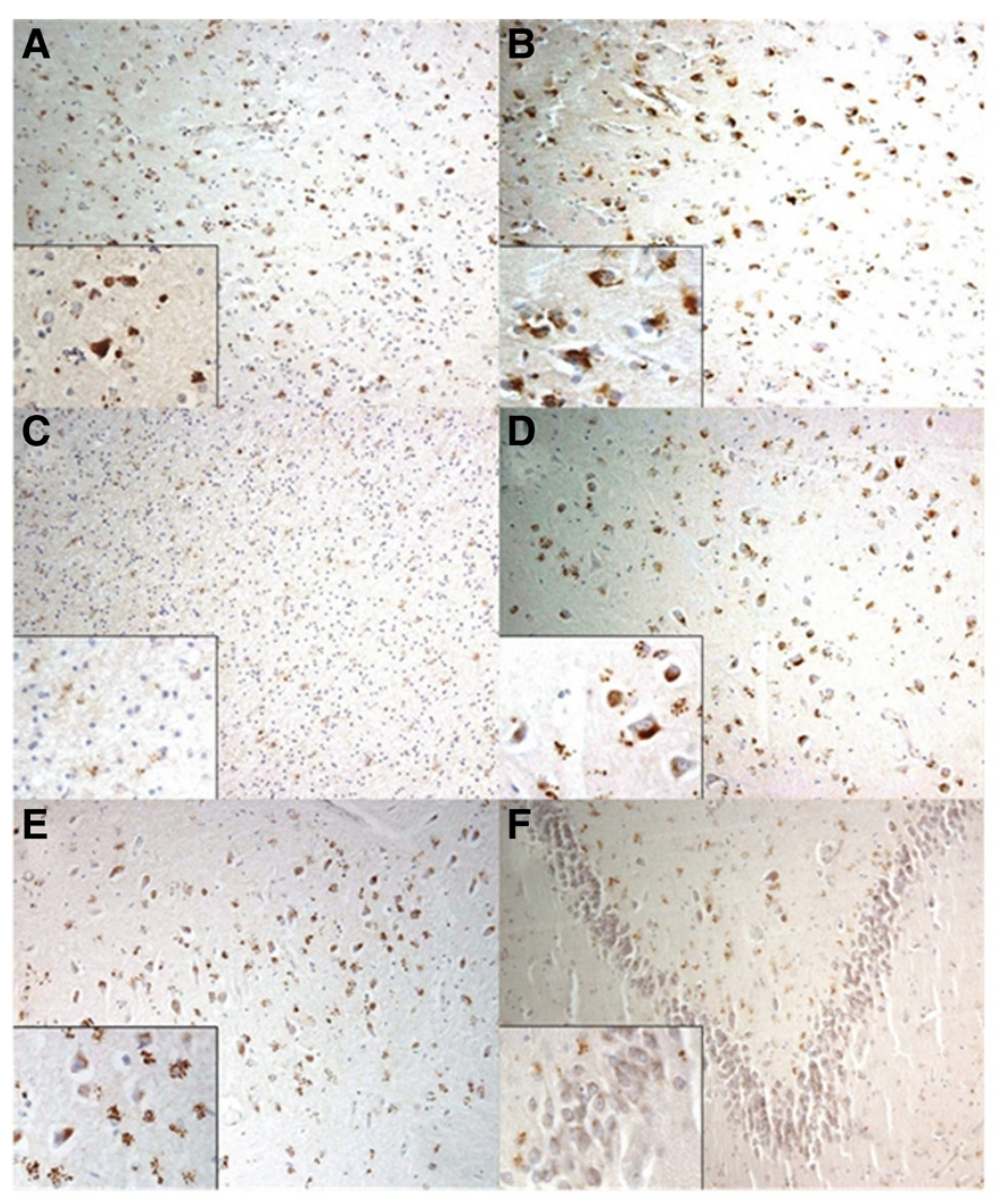

Figure 2 Characteristic HSP27-positive granules in the cytoplasm of neurons and glial cells of different regions of frontotemporal lobar degeneration cases under light microscopy. (A) Temporal cortex. (B) Frontal cortex. (C) White matter of cortex. (D) CA4 of hippocampus. (E) CA3 of hippocampus. (F) Dentate gyrus of hippocampus.

Post hoc analysis showed the proportion of cases affected was higher in FTLD subjects than in controls in all regions (temporal cortex: neurons, $P=0.008$; glial cells of grey matter, $P=0.039$; glial cells of white matter, $P=0.000$; frontal cortex: neurons, $P=0.000$; glial cells of grey matter, $P=0.000$; glial cells of white matter: $P=0.000$; hippocampus: glial cells of CA4, $P=0.000$ ). Similarly, there was a higher proportion of cases affected in FTLD than MND in glial cells of both grey matter $(P=0.000)$ and white matter $(P=0.000)$ of both the frontal and temporal cortices ( $P=0.000$ in every instance) and for glial cells of the hippocampus $(P=0.000)$.

There was a significantly higher proportion of cases affected in FTLD than in AD in respect of glial cells of both grey matter $(P=0.009)$ and white matter $(P=0.002)$ of the frontal cortex, and marginally so for glial cells of the hippocampus $(P=0.013)$. There was a higher proportion of cases affected in AD than in controls for the temporal cortex white matter glial cells $(P=0.012)$ and the frontal cortex neurons $(P=0.009)$. There were no significant differences between MND and control cases in any region, except for neurons in CA1 and subiculum $(P=0.001)$.

Subgroup analysis between FTLD-tau and FTLD-TDP groups, between FTLD-MAPT and FTLD-Pick groups, and between FTLD-TDP A, B and C groups all showed no significant differences in the frequencies of HSP27positive cases, except for comparison between FTLDTDP subtypes A, B and C for glial cells in white matter of the frontal cortex $\left(X^{2}=11.9, P=0.003\right)$, where a higher proportion of FTLD-TDP A cases $(P=0.005)$ were affected compared to FTLD-TDP $C$ cases.

The severity of HSP27 immunoreactivity in neurons and glial cells in the different brain regions in all four diagnostic groups was compared by Kruskal-Wallis test, with post hoc Mann-Whitney testing where significant. In general, analysis showed that neurons and glial cells in patients with AD and FTLD were more severely affected than patients with MND Q12 and control subjects (Figure 4). Comparisons of the FTLD (overall), AD, MND and control groups showed significant differences in the 


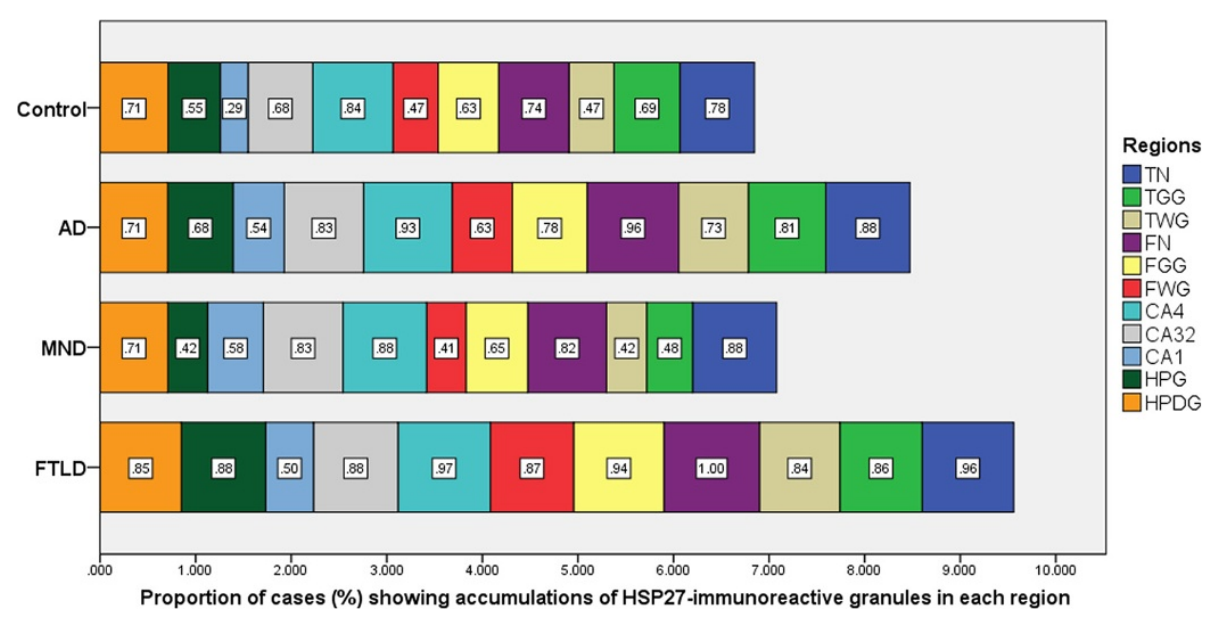

Figure 3 Proportion of cases with HSP27-positive granules in different brain regions in each group. AD, Alzheimer's disease; CA1, neurons of CA 1 + subiculum regions of the hippocampus; CA3/2, Neurons of CA3/2 region of the hippocampus; CA4, Neurons of CA4 region of the hippocampus; FGG, Grey matter glial cells of the frontal cortex; FN, Neurons of the frontal cortex; FTLD, Frontotemporal lobar degeneration; FWG, White matter glial cells of the frontal cortex; HPDG, Granular cells of the dentate gyrus of the hippocampus; HPG, Glial cells of the hippocampus; MND, Motor neuron disease; TGG, Grey matter glial cells of the temporal cortex; TN, Neurons of the temporal cortex; TWG, White matter glial cells of the temporal cortex.

severity of HSP27 rating values for neurons $\left(F_{(3,162)}=10.7\right.$, $P=0.013)$, grey matter glial cells $\left(F_{(3,162)}=21.7, P=0.000\right)$ and white matter glial cells $\left(F_{(3,162)}=25.8, P=0.000\right)$ of the temporal cortex, and for neurons $\left(F_{(3,151)}=18.9, P=0.001\right)$, grey matter glial cells $\left(F_{(3,151)}=29.2, P=0.000\right)$ and white matter glial cells $\left(F_{(3,151)}=46.0, P=0.000\right)$ of the frontal cortex. In the hippocampus, there were also significant differences in the severity of HSP27 rating for neurons of CA4 $\left(F_{(3,154)}=17.8, P=0.000\right)$ region and for glial cells $\left(F_{(3,154)}=14.9, P=0.002\right)$ and granule cells of dentate gyrus $\left(F_{(3,154)}=11.8, P=0.008\right)$.

Post hoc testing showed that there was significantly greater HSP27 immunostaining of neurons of the temporal cortex in FTLD compared to controls $(P=0.016)$ and MND $(P=0.007)$ groups, and marginally significantly greater staining in $\mathrm{AD}$ compared to $\mathrm{MND}(P=0.028)$. Similarly, there was significantly greater HSP27 immunostaining of glial cells of grey matter of the temporal cortex in AD compared to controls $(P=0.002)$.

In the frontal cortex, there was significantly greater HSP27 staining neurons of the frontal cortex in FTLD $(P=0.004)$ and AD groups $(P=0.001)$ compared to controls. There was also significantly greater HSP27 staining in glial cells of grey matter in FTLD compared to MND $(P=0.010)$, and in glial cells of the white matter in FTLD compared to both MND $(P=0.000)$ and $\operatorname{AD}(P=0.002)$ groups.

In the hippocampus, there was significantly greater HSP27 staining in CA4 region in the FTLD group $(P=0.000)$ and the AD group $(P=0.002)$ compared to controls, and in the FTLD group compared to the MND group $(P=0.009)$. There was also significantly greater
HSP27 staining in FTLD compared to AD $(P=0.030)$, control $(P=0.009)$ and MND $(P=0.001)$ for glial cells of the hippocampus, and for granule cells of dentate gyrus in FTLD compared to MND $(P=0.002)$ and control $(P=0.007)$.

However, subgroup analysis between FTLD-tau and FTLD-TDP groups, between FTLD-MAPT and FTLD-Pick groups, and between FTLD-TDP type A, B and C groups, all showed no significant differences in the severity of HSP27 rating values, except for significant differences between FTLD-tau and FTLD-TDP groups for grey matter glial cells of the frontal cortex $(P=0.006)$.

\section{Western blotting}

Western blotting of each fraction of the frontal cortex from the two samples showed two normal bands at approximately $50 \mathrm{kDa}$ and approximately $25 \mathrm{kDa}$, respectively (Figures 5D and 5E). Although there was higher HSP27 expression in FTLD cases than in control cases, this was without any change in banding patterns (Figures 5A, 5B and 5C). Where there were matched samples, there was good correlation between the intensity of staining on Western blots and the severity of HSP27 immunoreactive changes on immunohistochemical stains (Table 5). There were no obvious differences in HSP27 expression between the pathological subtypes in FTLD (Figures 5A, 5B and 5C).

\section{Discussion}

Although the physical and physiological relationships between chaperone proteins, such as HSP27, and misfolded proteins has been studied intensively, a role for 


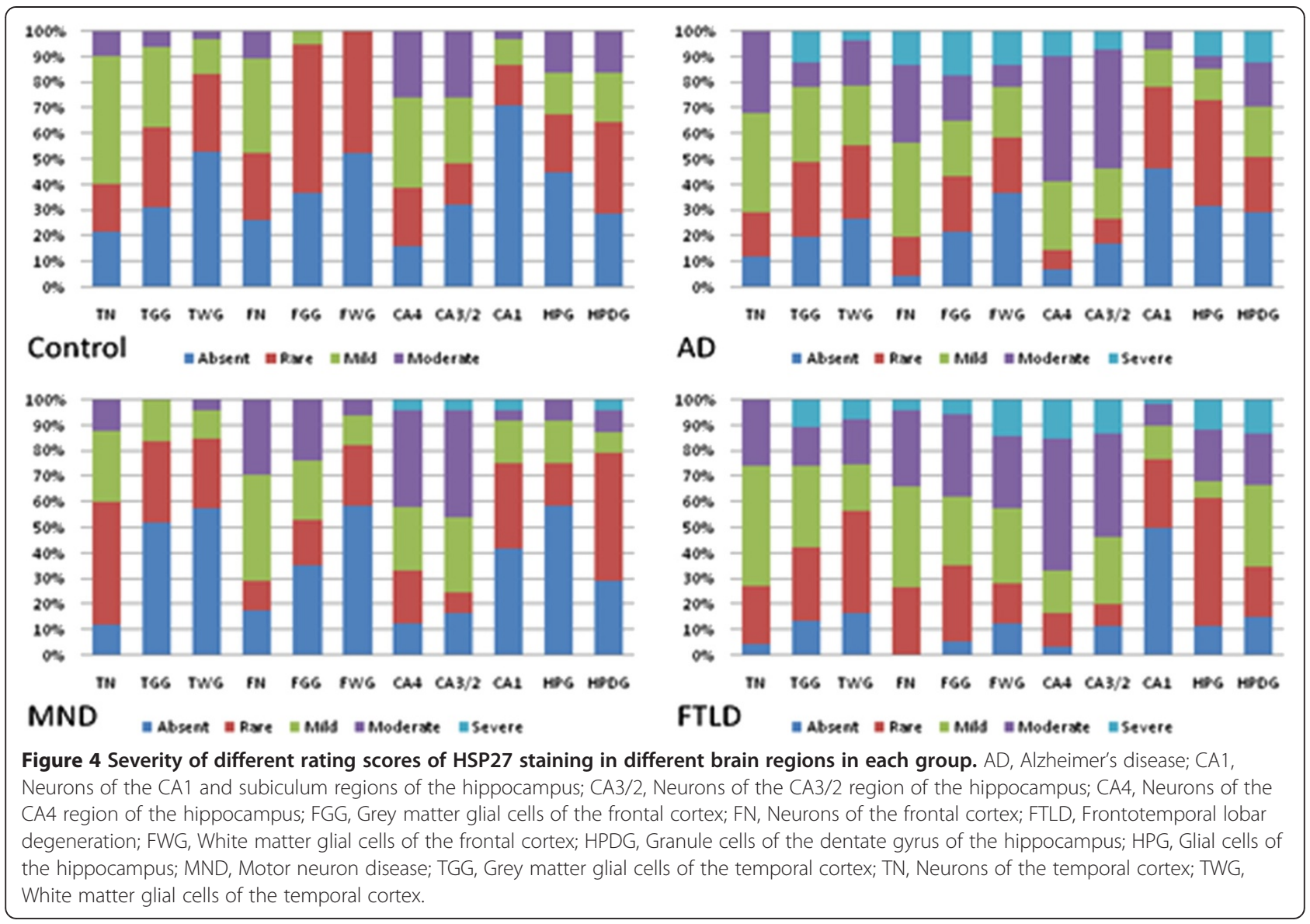

HSP27 in the pathogenesis of neurodegenerative disease is still incompletely understood. In the present study, the expression of HSP27 in FTLD, AD, MND and normal controls was analysed through immunohistochemical staining with HSP27 antibody and Western blotting. HSP27 is usually induced in response to cellular stress, but it can also stabilise the actin cytoskeleton, attenuate apoptosis, participate in cell differentiation and survival when phosphorylated [23], and promote cell cycle reentry into the S-phase by facilitating ubiquitination and degradation of the cell cycle inhibitor $\mathrm{p} 27^{\mathrm{Kip} 1}$ [24]. The affinity of HSP27 for proteins to be chaperoned is modulated by its phosphorylation and oligomerisation status [25].

Our present results show that the expression patterns of HSP27 in these neurodegenerative diseases are quite different. Patients with FTLD and AD showed numerous intensely immunostained, HSP27-positive granules in the cytoplasm of pyramidal neurons of the cerebral cortex, along with diffuse clusters of similar granules within glial cells. Such changes were particularly severe in the CA4 region of the hippocampus and in cells of the dentate gyrus. Subgroup analysis showed that there were no differences in the patterns of immunostaining between FTLD-tau and FTLD-TDP cases. However, in cases of MND and normal controls, we found less extensive accumulation of HSP27 immunoreactive granules in these same brain areas. These observations probably reflect the greater ongoing neurodegeneration in these brain areas in AD and FTLD compared to MND.

There have been few previous studies in which investigators have evaluated HSP27 reactivity in neurodegenerative disease, and then only in relationship to AD. To our knowledge, there have been no such studies in FTLD. In general, these studies have shown there to be an increase in expression of HSP27 in AD brain tissue or in immunolabelling of pathological structures [12,25-27]. Using a rabbit anti-HSP27 antiserum raised against a hybrid protein containing the $\mathrm{N}$-terminal part of the murine HSP27 (amino acids 1 to 110) and the C-terminal part of the human HSP27 (amino acids 111 to 208), Renkawek and co-workers showed a highly induced expression of HSP27 in many proliferating astrocytes in affected regions of cerebral cortex, especially in those areas rich in senile plaques [12]. They also reported neurofibrillary tangles, Hirano bodies and some hippocampal neurons to be immunopositive [12]. The expression of HSP27 correlated with the severity of $\mathrm{AD}$-specific morphological changes and the duration of dementia [12]. Shimura and co-workers 


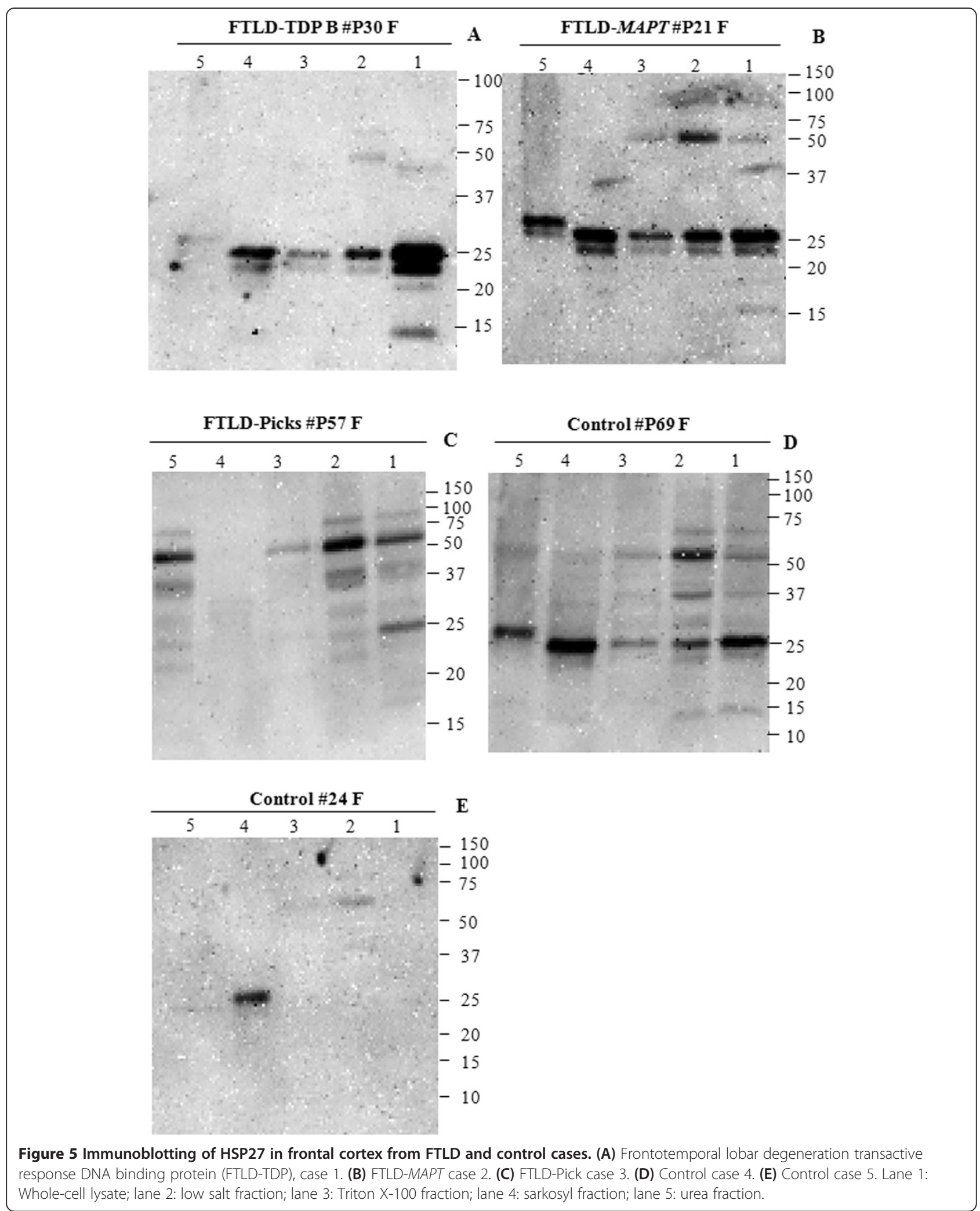

partially confirmed these findings, demonstrating a coimmunoprecipitation of HSP27 with hyperphosphorylated tau protein from $\mathrm{AD}$ brain, but not with nonphosphorylated tau from normal brain homogenates [25]. However, in another study, a diffuse, rather than granular, HSP27 immunostaining was observed in a subpopulation of astrocytes 
Table 5 Semiquantitative rating of immunoblotting of HSP27 and comparison with immunohistochemistry ${ }^{a}$

\begin{tabular}{|c|c|c|c|c|c|c|c|c|c|c|c|c|c|c|}
\hline \multirow[b]{2}{*}{ Case } & \multicolumn{5}{|c|}{ Approximately $25-\mathrm{kDa}$ band } & \multicolumn{5}{|c|}{ Approximately $50-\mathrm{kDa}$ band } & \multicolumn{4}{|c|}{ IHC rating scores } \\
\hline & $\overline{\mathrm{WL}}$ & LS & TX & SARK & UF & $\overline{\mathrm{WL}}$ & LS & TX & SARK & $\overline{\text { UF }}$ & $\overline{F-N}$ & F-GG & F-WG & F-Astro \\
\hline 1 & ++++ & ++ & + & ++ & $+/-$ & $+/-$ & $+/-$ & - & - & - & 3 & 3 & 2 & 3 \\
\hline 2 & +++ & +++ & ++ & +++ & +++ & + & ++ & + & - & - & 3 & 3 & 3 & 3 \\
\hline 3 & + & $+/-$ & - & - & $+/-$ & ++ & +++ & + & - & ++ & 2 & 3 & 4 & 4 \\
\hline 4 & +++ & ++ & + & +++ & ++ & $+/-$ & ++ & $+/-$ & - & $+/-$ & nd & nd & nd & nd \\
\hline 5 & - & - & - & ++ & - & - & $+/-$ & - & - & - & 1 & 1 & 1 & 2 \\
\hline
\end{tabular}

a + /++/+++ of bands; +/- very faint bands; - no bands at all; F-Astro, Astrocytes of frontal cortex; F-GG, Grey matter glial cells of frontal cortex; F-N, Neurons of frontal cortex; F-WG, White matter glial cells of frontal cortex; IHC, Immunohistochemistry; LS, Low salt fraction; nd, not done; SARK, Sarkosyl fraction; TX, Triton X-100 fraction; UF, Urea fraction; WL, Whole protein fractions.

and microglial cells associated with senile plaques and cerebral amyloid angiopathy, but this was not seen in neurofibrillary tangles in either the neocortex or hippocampus [27]. In a biochemical study, Bjorkdahl and colleagues reported the expression of HSP27 in AD brain to be increased (by approximately $20 \%$ ) in homogenates from the medial temporal cortex [26]. Moreover, this increase in HSP27 correlated significantly with the expression levels of the majority of the phosphorylated tau epitopes, but not with AT270, Alz-50 or AT100 [26].

In the present study, we observed numerous intensely immunostained, HSP27-positive granules in the cytoplasm of pyramidal neurons of the cerebral cortex and as diffuse clusters of similar appearing granules within glial cells, in both FTLD and AD. However, in AD, there was no HSP27 immunostaining of senile plaques or neurofibrillary tangles within the frontal and temporal cortices or within the hippocampus. The failure to confirm previous findings [12] (but see [27]) may be partly due to the different HSP27 antibodies used. In the present study, the antiHSP27 antibody used (ab5579; Abcam) is a rabbit polyclonal raised against a synthetic peptide, LLRGPSWDPFRC, corresponding to amino acids 10 to 21 of human HSP27. It is much more specific than the rabbit anti-HSP27 antiserum raised against a hybrid protein containing the $\mathrm{N}$-terminal part of the murine Hsp25 (amino acids 1 to 110) and the C-terminal part of the human HSP27 (amino acids 111 to 208) used by Renkawek et al. [12]. Hence, the staining of plaques (amyloid) or tangles with this latter antibody may represent nonspecific cross-reactivity, an interpretation consistent with findings reported by Wilhelmus and colleagues [27], who likewise failed demonstrate any immunostaining of these structures with HSP27. Our presently reported observations that no HSP27 immunostaining was seen in Pick bodies in patients with FTLD-tau, or in TDP-43 immunoreactive neuronal cytoplasmic inclusions in patients with FTLDTDP or MND, would support this conclusion. Interestingly, in previous studies where HSP27 immunostaining of astrocytes has been reported [12,27], this has taken the form of a homogeneous, diffuse cytoplasmic staining rather than one with pronounced granularity as demonstrated here. Again, different antibody specificities may be responsible, picking up HSP27 proteins either in a different conformational state or in aggregated forms.

The pattern of HSP27 immunoreactivity in cells may reflect an activation of protein quality control systems consequent upon damage to neurons or a response to the presence of accumulated tau/TDP-43 proteins. It is increasingly clear that the cooperation of proteolytic machineries, such as the proteasome and lysosome, and chaperone systems is required to prevent the aggregation of potentially toxic misfolded proteins. If such misfolded proteins escape appropriate refolding by chaperone systems, the proteasome and lysosome systems will be recruited to degrade them. However, if the production of such misfolded proteins overwhelms the capacity of the protein quality control systems, protein aggregates will form.

Within our control group, there was variability in the expression of HSP27. In some control subjects similar (to AD and FTLD), although less intense, HSP27 changes were present in the cytoplasm of pyramidal neurons of the cerebral cortex, and again as diffuse clusters of similar granules within glial cells. Such changes probably reflect the mild AD-type pathological changes present in such control subjects. In a recent study of patients with amnestic mild cognitive impairment (aMCI), a transitional stage between normal ageing and $\mathrm{AD}$, there was a significant increase (200\%) in the expression of HSP27 in the hippocampus in the aMCI group compared to controls [28], which would be consistent with these 'intermediate' histological findings.

It is presently unclear which cellular compartment or substructure might be associated with HSP27 immunostaining. The granularity of the staining suggests a phagocytotic location. Consequently, we stained sections in which there was an abundance of HSP27-immunoreactive granules with LAMP1 (a lysosomal marker) and LCA3 (a marker of autophagy) antibodies. LAMP1 detected granular structures in neurons and glia similar to those detected by HSP27, but none such were demonstrated 
in LCA3 immunostaining. These observations suggest that HSP27 immunoreaction is possibly associated with the cells' lysosomal compartments and may represent accumulated protein undergoing or awaiting degradation or the product of a failed degradation process. Nonetheless, a proteasomal location is also possible, and the pattern of immunoreactivity could represent proteasomes clogged or choked with accumulated protein. However, the fact that the 'granules' were not immunostained with either p62 or ubiquitin antibodies suggests that the HSP27 immunolabelled material does not contain either of these proteins, which act as molecular 'flags' for proteasomal degradation. Therefore, the subcellular localisation of HSP27 immunostaining, and its role in the neurodegenerative cascade, remains uncertain though may possibly be lysosomal.

\section{Conclusions}

In this study, we show that there are distinct patterns of HSP27 immunostaining with respect to different types of neurons or glial cells in different brain regions in patients with neurodegenerative disease. In general, patients with AD and FTLD were more severely affected than were patients with MND and control subjects; however, there was no association with FTLD histological subtype. Moreover, the 'classic' neuropathological changes in FTLD, $\mathrm{AD}$ and MND were not immunoreactive to HSP27, with the lack of HSP27 immunostaining of 'classic' pathology suggesting that HSP27 does not play a direct role in the formation of these structures. Therefore, HSP27 immunostaining in neurodegenerative disease likely reflects the extent of ongoing neurodegeneration in affected brain areas and is not specific to FTLD, $\mathrm{AD}$ or MND. It may represent an accumulation of misfolded, damaged or unwanted proteins, waiting for degradation via proteolytic mechanisms, though the precise pathway that this might enter, or fail to enter, for such a purpose remains unclear.

\section{Abbreviations \\ AD: Alzheimer's disease; A $\beta$ : Amyloid- $\beta$ protein; FTLD: Frontotemporal lobar degeneration; HSP: Heat shock protein; MND: Motor neuron disease; \\ PD: Parkinson's disease; TDP-43: Transactive response DNA binding protein 43.}

\section{Competing interests}

The authors declare that they have no competing interests.

\section{Authors' contributions}

XZ did all the immunohistochemistry and microscopy assessments and helped with writing the manuscript. JS helped with data analysis and writing the manuscript. AR and YD provided technical assistance as well as assistance with data acquisition. JT and DM designed and supervised the study, interpreted data, helped with microscopy assessments and wrote the manuscript. All authors read and approved the final manuscript, and all authors agree to be accountable for all aspects of the work in ensuring that questions related to the accuracy or integrity of any part of the work are appropriately investigated and resolved.

\section{Acknowledgements}

We acknowledge the support of Alzheimer's Research UK and the Alzheimer's Society through their funding of the Manchester Brain Bank under the Brains for Dementia Research (BDR) initiative. XZ received an Overseas Research Student Award from the University of Manchester. XZ, JS and JT were supported by the 111 Project (No.B08006), the Technological Platform of Clinical Evaluation and Research for New Herbal Medicinal Products (No.2011ZX09302-006-01) from the People's Republic of China, the Innovative Research Team in Beijing University of Chinese Medicine (No: 2011-CXTD-21) and the Study of Secondary Prevention with Chinese Herbal Medicine for Chronic Diseases (No.Z111107056811043).

\section{Author details}

${ }^{1}$ The Third Department of Neurology, Dongzhimen Hospital, Beijing University of Chinese Medicine, No. 5 Haiyuncang Street, Dongcheng District, Beijing 100700, China. ${ }^{2}$ Clinical and Cognitive Sciences Research Group, Institute of Brain, Behaviour and Mental Health, Faculty of Medical and Human Sciences, University of Manchester, Salford Royal Hospital, Salford M6 8HD, UK. ${ }^{3}$ Beijing University of Chinese Medicine Neurology Centre, Dongzhimen Hospital, Beijing University of Chinese Medicine, 5 Haiyuncang Street, Beijing 100700, People's Republic of China, China. ${ }^{4}$ Clinical and Cognitive Neuroscience Research Group, University of Manchester, Salford Royal Foundation NHS Trust, Salford, M6 8HD, UK.

Received: 17 December 2013 Accepted: 20 October 2014

Published online: 17 December 2014

\section{References}

1. Kopito RR, Ron D: Conformational disease. Nat Cell Biol 2000, 2:E207-E209.

2. Muchowski PJ, Wacker JL: Modulation of neurodegeneration by molecular chaperones. Nat Rev Neurosci 2005, 6:11-22.

3. Ciechanover A, Brundin P: The ubiquitin proteasome system in neurodegenerative diseases: sometimes the chicken, sometimes the egg. Neuron 2003, 40:427-446.

4. Vos MJ, Hageman J, Carra S, Kampinga HH: Structural and functional diversities between members of the human HSPB, HSPH, HSPA, and DNAJ chaperone families. Biochemistry 2008, 47:7001-7011.

5. Hartl FU: Molecular chaperones in cellular protein folding. Nature 1996, 381:571-580.

6. Hartl FU, Hayer-Hartl M: Converging concepts of protein folding in vitro and in vivo. Nat Struct Mol Biol 2009, 16:574-581.

7. Landry J, Huot J: Modulation of actin dynamics during stress and physiological stimulation by a signaling pathway involving p38 MAP kinase and heat-shock protein 27. Biochem Cell Biol 1995, 73:703-707.

8. Jakob U, Gaestel M, Engel K, Buchner J: Small heat shock proteins are molecular chaperones. J Biol Chem 1993, 268:1517-1520.

9. Haslbeck M, Miess A, Stromer T, Walter S, Buchner J: Disassembling protein aggregates in the yeast cytosol: the cooperation of Hsp26 with Ssa1 and Hsp104. J Biol Chem 2005, 280:23861-23868.

10. Garrido C, Bruey JM, Fromentin A, Hammann A, Arrigo AP, Solary E: HSP27 inhibits cytochrome $c$-dependent activation of procaspase-9. FASEB J 1999, 13:2061-2070.

11. Bruey J-M, Ducasse C, Bonniaud P, Ravagnan L, Susin SA, Diaz-Latoud C, Gurbuxani S, Arrigo AP, Kroemer G, Solary E, Garrido C: Hsp27 negatively regulates cell death by interacting with cytochrome c. Nat Cell Biol 2000, 2:645-652.

12. Renkawek K, Bosman G, de Jong W: Expression of small heat-shock protein hsp 27 in reactive gliosis in Alzheimer disease and other types of dementia. Acta Neuropathol (Berl) 1994, 87:511-519.

13. Neary D, Snowden JS, Gustafson L, Passant U, Stuss D, Black S, Freedman M, Kertesz A, Robert PH, Albert M, Boone K, Miller BL, Cummings J, Benson DF: Frontotemporal lobar degeneration: a consensus on clinical diagnostic criteria. Neurology 1998, 51:1546-1554.

14. Rascovsky K, Hodges JR, Knopman D, Mendez MF, Kramer JH, Neuhaus J, van Swieten JC, Seelaar H, Dopper EGP, Onyike CU, Hillis AE, Josephs KA, Boeve BF, Kertesz A, Seeley WW, Rankin KP, Johnson JK, Gorno-Tempini ML, Rosen H, Prioleau-Latham CE, Lee A, Kipps CM, Lillo P, Piguet O, Rohrer JD, Rossor MN, Warren JD, Fox NC, Galasko D, Salmon DP, et al: Sensitivity of revised diagnostic criteria for the behavioural variant of frontotemporal dementia. Brain 2011, 134:2456-2477. 
15. Cairns NJ, Bigio EH, Mackenzie IRA, Neumann M, Lee VMY, Hatanpaa KJ, White CL 3rd, Schneider JA, Grinberg LT, Halliday G, Duyckaerts C, Lowe JS, Holm IE, Tolnay M, Okamoto K, Yokoo H, Murayama S, Woulfe J, Munoz DG, Dickson DW, Ince PG, Trojanowski JQ, Mann DMA: Neuropathologic diagnostic and nosologic criteria for frontotemporal lobar degeneration: consensus of the Consortium for Frontotemporal Lobar Degeneration. Acta Neuropathol 2007, 114:5-22.

16. Mackenzie IRA, Neumann M, Baborie A, Sampathu DM, Du Plessis D, Jaros E, Perry RH, Trojanowski JQ, Mann DMA, Lee VMY: A harmonized classification system for FTLD-TDP pathology. Acta Neuropathol (Berl) 2011, 122:111-113.

17. Brooks BR, Miller RG, Swash M, Munsat TL: El Escorial revisited: revised criteria for the diagnosis of amyotrophic lateral sclerosis. Amyotroph Lateral Scler Other Motor Neuron Disord 2000, 1:293-299.

18. McKhann G, Drachman D, Folstein M, Katzman R, Price D, Stadlan EM: Clinical diagnosis of Alzheimer's disease: report of the NINCDS-ADRDA Work Group under the auspices of Department of Health and Human Services Task Force on Alzheimer's Disease. Neurology 1984, 34:939-944.

19. Mirra SS, Heyman A, McKeel D, Sumi SM, Crain BJ, Brownlee LM, Vogel FS, Hughes JP, van Belle G, Berg L: The Consortium to Establish a Registry for Alzheimer's Disease (CERAD): Part II. Standardization of the neuropathologic assessment of Alzheimer's disease. Neurology 1991, 41:479-486.

20. Davidson Y, Amin H, Kelley T, Shi J, Tian J, Kumaran R, Lashley T, Lees AJ, Du Plessis D, Neary D, Snowden J, Akiyama H, Arai T, Hasegawa M, Bandopadhyay R, Sikkink S, Pickering-Brown S, Mann DM: TDP-43 in ubiquitinated inclusions in the inferior olives in frontotemporal lobar degeneration and in other neurodegenerative diseases: a degenerative process distinct from normal ageing. Acta Neuropathol (Berl) 2009, 118:359-369.

21. Davidson Y, Kelley T, Mackenzie IRA, Pickering-Brown S, Du Plessis D, Neary D, Snowden J, Mann DA: Ubiquitinated pathological lesions in frontotemporal lobar degeneration contain the TAR DNA-binding protein, TDP-43. Acta Neuropathol (Berl) 2007, 113:521-533.

22. Neumann M, Sampathu DM, Kwong LK, Truax AC, Micsenyi MC, Chou TT, Bruce J, Schuck T, Grossman M, Clark CM, McCluskey LF, Miller BL, Masliah E, Mackenzie IR, Feldman H, Feiden W, Kretzschmar HA, Trojanowski JQ, Lee VM: Ubiquitinated TDP-43 in frontotemporal lobar degeneration and amyotrophic lateral sclerosis. Science 2006, 314:130-133.

23. Kostenko S, Moens U: Heat shock protein 27 phosphorylation: kinases, phosphatases, functions and pathology. Cell Mol Life Sci 2009, 66:3289-3307.

24. Parcellier A, Brunet M, Schmitt E, Col E, Didelot C, Hammann A, Nakayama K Nakayama KI, Khochbin S, Solary E, Garrido C: HSP27 favors ubiquitination and proteasomal degradation of p27Kip1 and helps S-phase re-entry in stressed cells. FASEB J 2006, 20:1179-1181.

25. Shimura H, Miura-Shimura Y, Kosik KS: Binding of tau to heat shock protein 27 leads to decreased concentration of hyperphosphorylated tau and enhanced cell survival. J Biol Chem 2004, 279:17957-17962.

26. Björkdahl C, Sjögren MJ, Zhou X, Concha H, Avila J, Winblad B, Pei JJ: Small heat shock proteins $\mathrm{Hsp} 27$ or aB-crystallin and the protein components of neurofibrillary tangles: Tau and neurofilaments. J Neurosci Res 2008, 86:1343-1352.

27. Wilhelmus MMM, Otte-Höller I, Wesseling P, De Waal RMW, Boelens WC, Verbeek MM: Specific association of small heat shock proteins with the pathological hallmarks of Alzheimer's disease brains. Neuropathol Appl Neurobiol 2006, 32:119-130.

28. Di Domenico F, Sultana R, Tiu GF, Scheff NN, Perluigi M, Cini C, Butterfield DA: Protein levels of heat shock proteins 27, 32, 60, 70, 90 and thioredoxin-1 in amnestic mild cognitive impairment: an investigation on the role of cellular stress response in the progression of Alzheimer disease. Brain Res 2010, 1333:72-81.

\section{doi:10.1186/s13195-014-0078-x}

Cite this article as: Zhang et al:: Expression of one important chaperone protein, heat shock protein 27 , in neurodegenerative diseases.

Alzheimer's Research \& Therapy 2014 6:78.

\section{Submit your next manuscript to BioMed Central and take full advantage of:}

- Convenient online submission

- Thorough peer review

- No space constraints or color figure charges

- Immediate publication on acceptance

- Inclusion in PubMed, CAS, Scopus and Google Scholar

- Research which is freely available for redistribution 